\title{
Torque characteristic of SI engine in dynamic operating states
}

\begin{abstract}
The article presents torque characteristic of the engine in dynamic operating conditions as a function of engine speed and throttle opening angle. All mentioned parameters are analyzed as independent variables over time. To develop such a characteristic an artificial neural network is used. The training data were obtained from measurements carried out on the test bench on SI engine. The operating states reflect all possible configurations of these parameters, which may occur during use of the vehicle in real traffic conditions. The article shows design of an artificial neural network that allows to designate the required dependences. Moreover, it describes the fit of the model to the measurement data, which clearly indicates its correctness. Then the developed characteristic in dynamic states is compared with the characteristic in static working states. The differences between them for selected cases of engine operation states are presented. It shows the versatility of the presented method.
\end{abstract}

Key words: engine torque, dynamic states, engine characteristic, artificial neural network

\section{Introduction}

Internal combustion engines used to propel automotive vehicles work mainly under dynamic working states. Static working states occur very rarely, primarily on idle or during extra-urban driving with constant speed. Therefore all engine characteristics should be based on measurements in dynamic working states. Currently manufactured measurement equipment enables such measurements but the problem is in data analysis. It is due to the fact that in dynamic working states engine parameters cannot be considered as a function of state. It is necessary to take into account engine parameters from previous time intervals. It leads to the situation where several values of few parameters affect another parameter.

Hence measurements in static states are commonly performed. On a basis of such measurements engine characteristics are determined and then saved in ECU. One of such characteristics is engine torque characteristic that in static states is a function of engine speed and throttle opening angle.

There are many methods that allow to designate engine torque characteristic. Except the one described above, there are also other ways. Some of them are as follows:

- the use of ANN to obtain a mathematical model of engine [4, 6-8],

- the use of nonlinear models based on mean-values algorithms [4, 5].

Dynamic states in combustion engines are very difficult to analyze because engine torque is strongly influenced by several factors: changes in kinetic energy in moving parts (e. g. crankshaft, camshafts or flywheel), unstable airflow in manifold or changes in linear speed of coolant or oil.

Due to the above problems, that are impossible to include, it was assumed in the article, that the engine is a black box. Thus only input (throttle angle and engine speed) and output (engine torque) parameters were measured in dynamic and static states and then analyzed in time intervals.

The tests were performed on SI engine with a displacement of $899 \mathrm{~cm}^{3}$ and nominal power of $29 \mathrm{~kW}$ at $5500 \mathrm{rpm}$. In the performed tests both dynamic and static characteristics were collected. Measurement methodology was described in details by the author in works $[2,3]$. Then these data are analyzed with use on ANN trained in supervised mode.

\section{Engine torque characteristic in static states}

\subsection{Engine test bed measurements}

In case of standard characteristic in static states measurements are performed on conventional eddy current engine dynamometer that ensures proper accuracy. Parameters such as engine speed, engine torque, throttle opening angle, air-fuel ratio etc. are constant. Engine inertial elements have then no influence because angular acceleration equals zero and throttle position is constant what ensures stable airflow in manifold.



Fig. 1. A single measurement in static state

A single exemplary measurement, lasting $20 \mathrm{~s}$, is shown in Fig. 1. Engine parameters are presented as originally measured - in Volts.

\subsection{Data analysis}

In case of characteristic based on static states data analysis is easy. To create such a $3 \mathrm{~d}$ characteristic, where:

$$
M=f(n, \alpha)
$$

only basic computational tools are needed e.g. 3d graph in Excel or cftool in Matlab. In this study the second method was used. A characteristic obtained for an engine, that tests were performed on, is shown in Fig. 2. It was created based on 80 measurements in static states. 


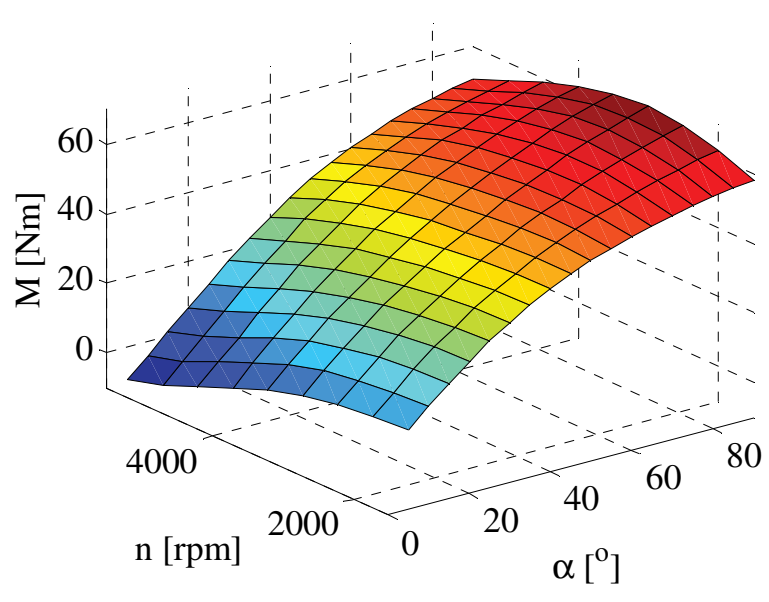

Fig. 2. Engine torque characteristic in static states

It can be noticed that for throttle angle of $90^{\circ}$ the characteristic is the same as external speed characteristic provided by the manufacturer.

\subsection{Conclusions}

Although such an engine torque characteristic is easy to designate, it gives only a rough dependency between engine torque vs rotational speed and throttle opening angle. It cannot be used in accurate calculations of dynamic transitions between two points on characteristic.

\section{Engine torque characteristic in dynamic states}

\subsection{Engine test bed measurements}

Measurements in dynamic states must be performed on the engine test bed that ensures rapid changes of both drag torque and throttle opening angle. In case of presented characteristic 3 engine parameters must be constantly measured: rotational speed, throttle opening angle and engine torque. A single measurement is shown in Fig. 3.

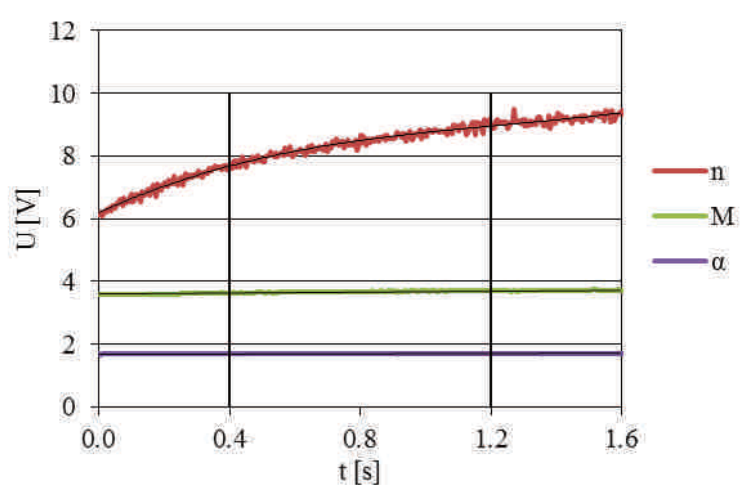

Fig. 3. Engine parameters in dynamic states

The graph clearly shows that in dynamic working states engine torque cannot be considered as a state function of engine torque and throttle opening angle because in this case:

$$
M\left(t_{1}\right)=M\left(t_{2}\right)
$$

but:

$$
\mathrm{n}\left(\mathrm{t}_{1}\right) \neq \mathrm{n}\left(\mathrm{t}_{2}\right)
$$

As results from this example in dynamic states the engine torque cannot be considered as a state function of rotational speed and throttle opening angle:

$$
\mathrm{M} \neq \mathrm{f}(\mathrm{n}, \alpha)
$$

Parameters must be analyzed in time interval of specified length. ANN allows for such an analysis, what is described below.

\subsection{Data analysis with use of ANN}

Pursuant to information mentioned in the introduction, the analysis requires multidimensional attempt. Such an approach is possible with use of ANN method. Network can have many inputs that represent subsequent values of parameters in determined time interval because only then inertia phenomena can be included. It requires detailed analysis of time intervals. Moreover, it is impossible to determine up front which ANN architecture is the best for this particular problem. Therefore several configurations were examined before final choice.

All the data described in a previous point create a training sets for ANN. Before training, data were mixed, like described in [3], which improves training process significantly. Training set was constructed based on 220 measurements in dynamic states and 80 measurements in static states.

The first step is to decide which ANN architecture is the best for this particular problem. To do this, several network configurations were checked. In order to obtain relevant results each of 8 networks was trained with the same algorithm and the number of hidden neurons was always the same. According to recommendations included in [1-3] Lavenberg-Marquardt training algorithm and 10 hidden neurons were implemented.

Fig. 4 presents a representative dynamic state with described engine parameters that are later used in Table 1.

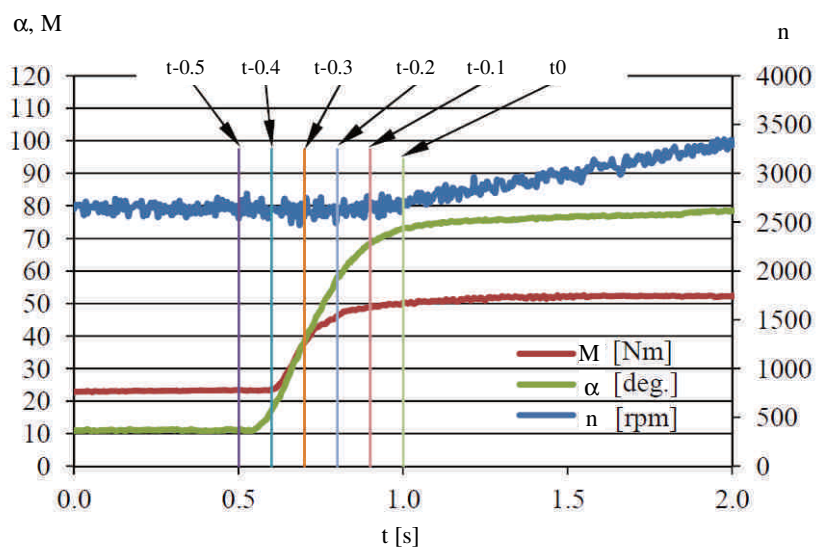

Fig. 4. Designation of time moments during engine operation

Author considered 8 ANN configurations (Table 1). The first one is in fact a static characteristic due to only one input of engine speed and one input of throttle opening angle. The next ones (2-8) ANNs take into account inertia phenomena and differ with number of inputs and time intervals, which are in the range 0-0.5 s. 
Table 1. Considered configurations of ANN architectures and corresponding mse and $R$ values

\begin{tabular}{|c|c|c|}
\hline No & ANN configuration & $\begin{array}{c}\text { Data fit after } \\
\text { training process } \\
x \text { axis }- \text { target } \\
y \text { axis }- \text { net output }\end{array}$ \\
\hline 1 & $\begin{array}{l}n\left(t_{0}\right) \\
\alpha\left(t_{0}\right)\end{array}$ & : \\
\hline 2 & $\begin{array}{l}\mathrm{n}\left(\mathrm{t}_{0}\right) \\
\mathrm{n}\left(\mathrm{t}_{-0,1}\right) \\
\alpha\left(\mathrm{t}_{0}\right) \\
\alpha\left(\mathrm{t}_{-0,1}\right)\end{array}$ & 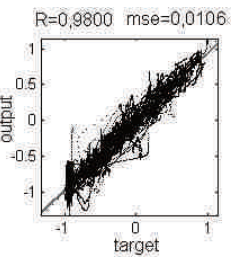 \\
\hline 3 & $\begin{array}{c}\mathrm{n}\left(\mathrm{t}_{0}\right) \\
\mathrm{n}\left(\mathrm{t}_{-0,2}\right) \\
\alpha\left(\mathrm{t}_{0}\right) \\
\alpha\left(\mathrm{t}_{-0,2}\right)\end{array}$ & target \\
\hline 4 & $\begin{array}{c}\mathrm{n}\left(\mathrm{t}_{0}\right) \\
\mathrm{n}\left(\mathrm{t}_{-0,3}\right) \\
\alpha\left(\mathrm{t}_{0}\right) \\
\alpha\left(\mathrm{t}_{-0,3}\right)\end{array}$ & target \\
\hline 5 & $\begin{array}{c}n\left(\mathrm{t}_{0}\right) \\
\mathrm{n}\left(\mathrm{t}_{-0,4}\right) \\
\alpha\left(\mathrm{t}_{0}\right) \\
\alpha\left(\mathrm{t}_{-0,4}\right)\end{array}$ & $\underbrace{\mathrm{R}=0,9803 \mathrm{mse}=0,0105}_{-1}$ \\
\hline 6 & $\begin{array}{c}\mathrm{n}\left(\mathrm{t}_{0}\right) \\
\mathrm{n}\left(\mathrm{t}_{-0,5}\right) \\
\alpha\left(\mathrm{t}_{0}\right) \\
\alpha\left(\mathrm{t}_{-0,5}\right)\end{array}$ & : \\
\hline 7 & $\begin{array}{l}\mathrm{n}\left(\mathrm{t}_{0}\right) \\
\mathrm{n}\left(\mathrm{t}_{-0,1}\right) \\
\mathrm{n}\left(\mathrm{t}_{-0,2}\right) \\
\alpha\left(\mathrm{t}_{0}\right) \\
\alpha\left(\mathrm{t}_{-0,1}\right) \\
\alpha\left(\mathrm{t}_{-0,2}\right)\end{array}$ & : \\
\hline 8 & $\begin{array}{c}\mathrm{n}\left(\mathrm{t}_{0}\right) \\
\mathrm{n}\left(\mathrm{t}_{-0,2}\right) \\
\mathrm{n}\left(\mathrm{t}_{-0,4}\right) \\
\alpha\left(\mathrm{t}_{0}\right) \\
\alpha\left(\mathrm{t}_{-0,2}\right) \\
\alpha\left(\mathrm{t}_{-0,4}\right)\end{array}$ & - \\
\hline
\end{tabular}

The best results (the smallest values of $m s e$ and $R$ ) are obtained with ANN number 8 from Table 1. It turns out that the best solution is obtained when there are 6 network inputs: three engine speeds and three throttle angles in time intervals of 0.2 second. The chosen network is shown in Fig. 5.

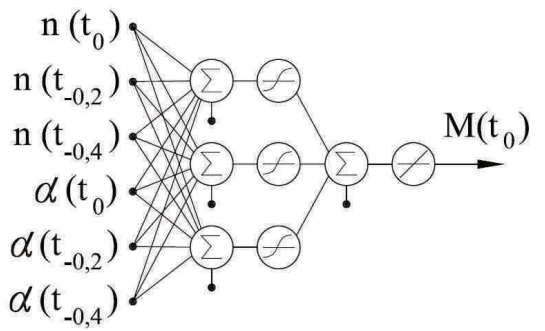

Fig. 5. ANN architecture that ensures best data approximation

The choice of proper ANN architecture is the first step. Then accurate number of hidden layers, number of hidden neurons and training algorithm must be chosen.

The number of hidden layers was set to one. According to [1] one layer with adequate number of neurons is capable of approximating any nonlinear problem with expected accuracy. Because training set consist of 210000 columns, Levenberg-Marquardt method was implemented [1,3]. As a result from performed calculations it turned out that 6 neurons is the best in this case. Further increase of neurons does not give any positive effect and the risk of overfitting raises.



Fig. 6. ANN data fit after training process

Finally obtained results are presented in Fig. 6 and Fig. 7. The graphs clearly show that ANN model adequately approximates measurement data. $\mathrm{R}$ value equals 0.98 and the mse $=0.00625$.



Fig. 7. Graph of errors for each subset during the ANN training 


\subsection{Conclusions}

Calculations performed with ANN show that this method can be used to analyze measurement data from dynamic states to design engine torque characteristic. ANN model fits measurement data very well, what is supported by values of mse, $\mathrm{R}$ parameter and correctness of obtained graphs from simulations shown in the next points. Engine torque is supposed to change in a predictable way. Some training processes generated such a model that was, because of this reason, obviously judged as improper. Such a situation is due to the fact, that initial values of weights and biases are random and even with the same ANN parameters, reasonable network is obtained only after series of training processes.

\section{Comparison of characteristic in dynamic states with characteristic in static states}

\subsection{Error definition}

In this chapter the engine torque characteristic designated with use of ANN will be compared to the static characteristic. The difference between values calculated by these characteristics is defined as follows:

$$
\delta=\frac{M_{\text {stat }}-M_{\text {dyn }}}{M_{\text {stat }}} \cdot 100 \%
$$

where: $\mathbf{M}_{\text {stat }}$ - engine torque calculated with use of characteristic in static states [Nm] (Fig. 2), $\mathbf{M}_{\text {dyn }}$ - engine torque calculated with use of characteristic in dynamic states $[\mathrm{Nm}]$ (designated with use of ANN).

In the article only situations with increasing engine speed will be considered therefore the error $\delta$ will always be positive.

\subsection{Increase of both engine speed and torque}

This simulation describes typical situation, when during driving with constant speed throttle is suddenly opened, engine torque raises rapidly and the vehicle begins to accelerate. In such case engine crankshaft angular acceleration depends on gear selected, slope of the road and vehicle speed. The bigger the transmission ratio (the lowest gear) the greater the engine angular acceleration.

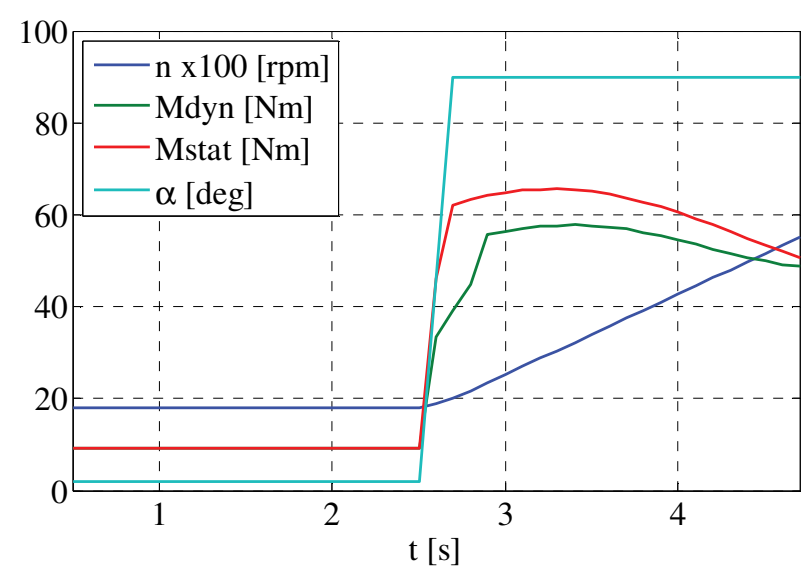

Fig. 8. Engine parameters in time of 2 seconds after full throttle opening
For the extreme case shown in Fig. 8 the difference in engine torque while increasing rotational speed is almost $15 \%$. What is also important, the torque increase while throttle opening is noticeably slower in comparison to static characteristic. As it can be resulted from Fig. 9 that the largest error is in range that is mostly often used in a real traffic (2000-3000 rpm) and decreases with engine speed raise. The difference between two characteristics becomes negligible with angular acceleration of about $20 \mathrm{rad} / \mathrm{s}^{2}$.

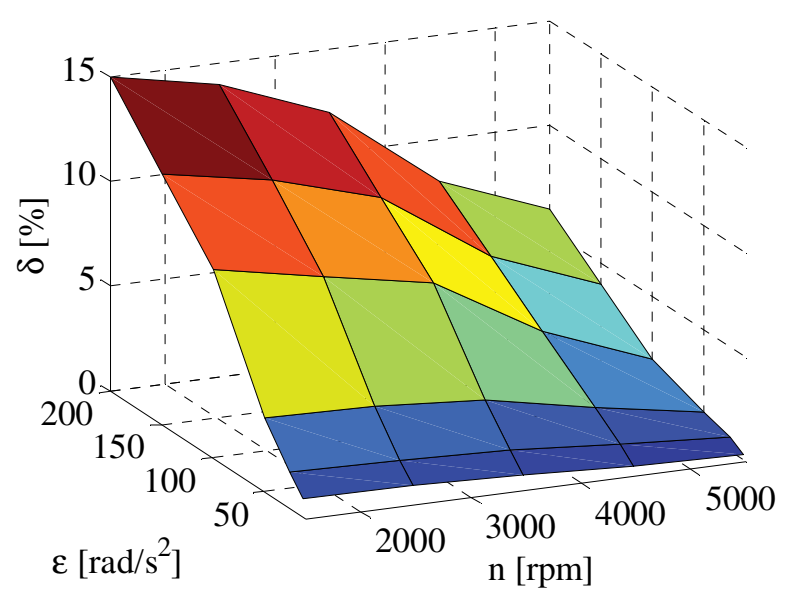

Fig. 9. Error $\delta$ in function of engine speed $n$ and angular acceleration $\varepsilon$ for throttle opening $90^{\circ}$

Fig. 10 shows similar situation but in this case throttle angle is only $30^{\circ}$. Maximum difference is for crankshaft angular acceleration of $200 \mathrm{rad} / \mathrm{s}^{2}$ and equals $10 \%$. It means that the difference $\delta$ decreases with decreasing throttle opening and is the largest at full throttle operation.

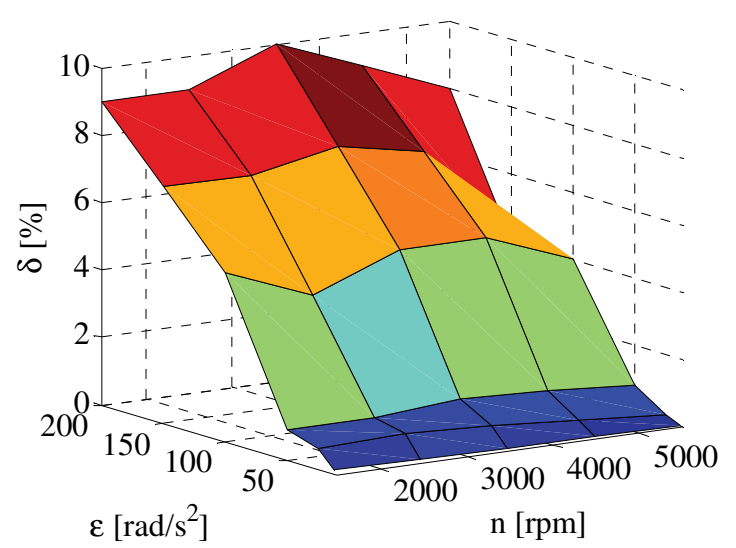

Fig. 10. Error $\delta$ in function of engine speed $n$ and angular acceleration $\varepsilon$ for throttle opening $30^{\circ}$

When angular acceleration is about $50 \mathrm{rad} / \mathrm{s}^{2}$ the difference becomes negligible.

\subsection{Increase in torque with constant speed}

Another situation that might occur on the road is the need to maintain constant speed while traffic resistances are growing up. In such cases throttle openings are usually much slower than in case of sudden acceleration. 


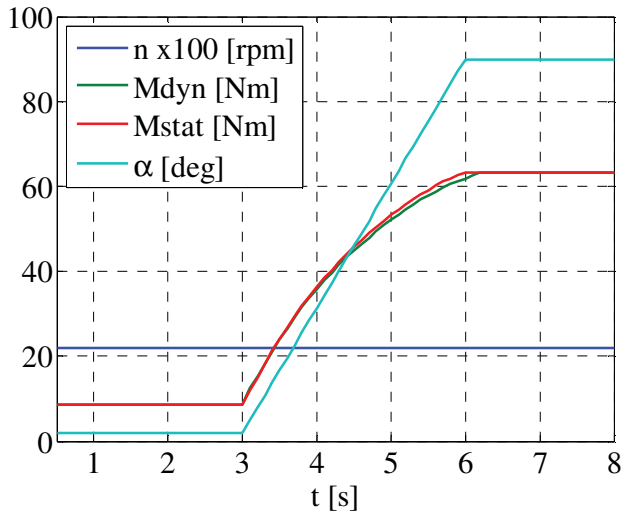

Fig. 11. Increase in engine torque with constant speed while full throttle opening

Fig. 11 clearly shows that there is a very small difference between values calculated by characteristics in static and dynamic states. Fig. 12 shows that the difference $\delta$ is the largest in the range of small engine speed and equals about $4 \%$. Then decreases with increasing engine speed.

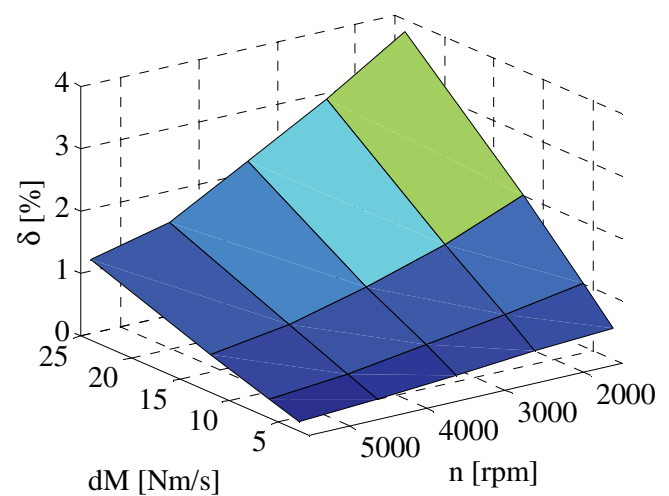

Fig. 12. Error $\delta$ in function of constant engine speed $\mathrm{n}$ and torque increase $\mathrm{dM}$

4.4. Influence of throttle opening time on engine torque

Another problem that occurs in dynamic operating states is delay in engine response to a control signal.

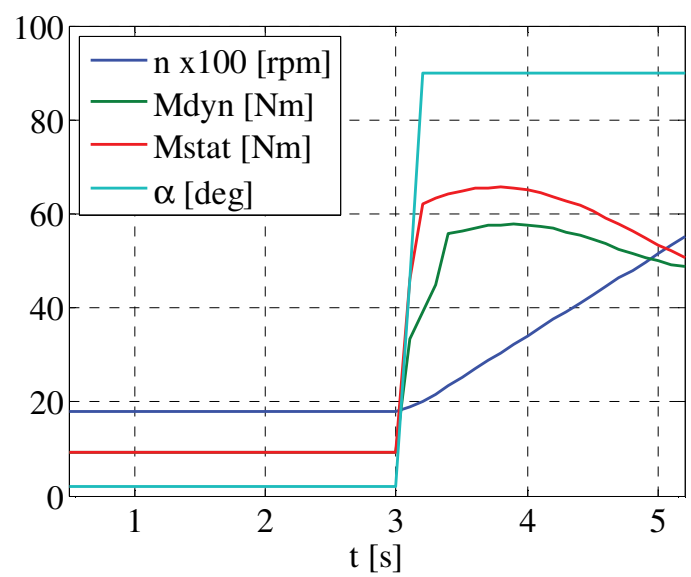

Fig. 13. Engine parameters during full throttle opening lasting $0.2 \mathrm{~s}$
It can be noticed (Fig. 13) that throttle opening lasts $0.2 \mathrm{~s}$ whereas engine torque reaches top value after $0.4 \mathrm{~s}$. So in this case delay is $100 \%$. It would be even more noticeable in turbocharged engines. During this time engine torque is even $30 \%$ smaller than it would result from static characteristic.

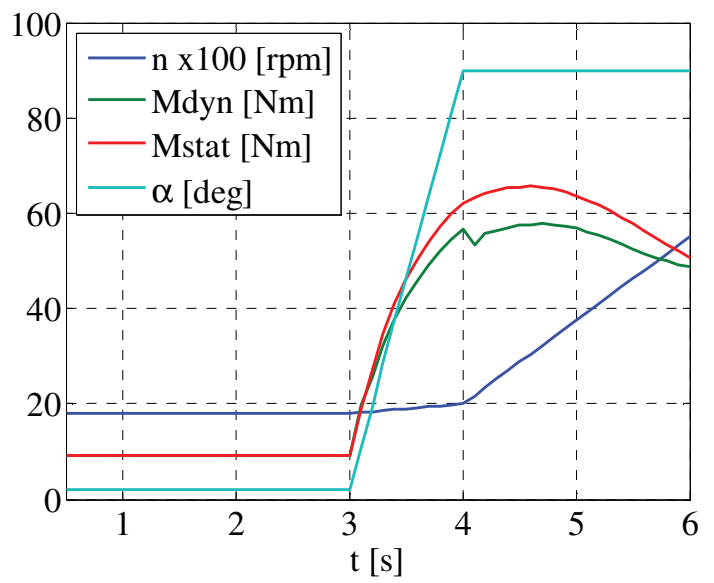

Fig. 14. Engine parameters during full throttle opening lasting $1 \mathrm{~s}$

In Fig. 14 full throttle opening is realized in $1 \mathrm{~s}$. Engine torque rises more slowly and the difference between two characteristics is smaller and equals $11 \%$. So the conclusion is that the more sudden throttle openings are the bigger differences between characteristics become. Because in a city traffic throttle operation is usually realized in a very short time, only characteristic in dynamic states should be used to obtain reliable results e.g. in simulations.

\section{Summary and conclusions}

The article shows that combustion engine torque characteristic in dynamic working states can be successfully designated with use of proper approach to the problem and with use of proper mathematical analysis. The ANN method allows for taking into consideration few following engine parameters in consecutive moments. Obtained model was verified in three ways: mse error, $\mathrm{R}$ parameter and simulations in point 4 .

Conducted simulations show that the greatest influence on differences between torque values calculated with use of static and dynamic characteristic has crankshaft angular acceleration. When engine speed raises rapidly, with angular acceleration of about $200 \mathrm{rad} / \mathrm{s}^{2}$, the engine torque is up to $15 \%$ smaller, than it would result from static torque characteristic. The difference between two characteristics decreases both with decreasing angular acceleration and throttle opening angle and becomes negligible with crankshaft acceleration of about $20 \mathrm{rad} / \mathrm{s}^{2}$.

The next conclusion is that in case of torque changes that occur at constant engine speed there is a very small difference between two characteristics. In such cases, regardless of throttle opening angle and engine speed the difference is smaller than $4 \%$.

Another conclusion from performed simulations is that in case of rapid full throttle opening lasting $0.2 \mathrm{~s}$ engine torque raises to top value in time of $0.4 \mathrm{~s}$. Which is quite 
much delay in a naturally aspirated engine. In such transient states the use of static characteristic instead of characteristic in dynamic states would result in a very big error up to $30 \%$.

\section{Nomenclature}

$\alpha \quad$ throttle opening angle

ANN artificial neural network

$\mathrm{n} \quad$ engine speed

$\mathrm{M}$ engine torque mse mean square error

$\mathrm{V} \quad$ vehicle speed

SI spark ignition

$\varepsilon \quad$ crankshaft angular acceleration

\section{Bibliography}

[1] BEALE, M.H., HAGAN, M.T., DEMUTH, H.B. Neural network toolbox. For use with Matlab. Release 2012b. www.mathworks.com. 2012.

[2] BERA, P. Fuel consumption analysis in dynamic states of the engine with use of artificial neural network. Combustion Engines. 2013, 162(4), 726-731.

[3] BERA, P. The use of artificial neural networks trained in supervised mode to the analysis of measurement data of combustion engines and automotive vehicles. Silniki Spalinowe $i$ Ekologia. 2014, Cracow University of Technology Press, 193-204.

[4] ISERMANN, R. Engine modeling and control. Modeling and Electronic Management of Internal Combustion Engines. Springer. 2014.

Piotr Bera, DEng. - AGH University of Science and Technology, Faculty of Mechanical Engineering and Robotics.

e-mail:PBera@agh.edu.pl
[5] KANG, M., ALAMIR, M., SHEN, T. Nonlinear constrained torque control for gasoline engines. IFAC-PapersOnLine. 2016, 49-18, 784-789.

[6] SERIKOV, S.A. Neural network model of internal combustion engine. Cybernetics and System Analysis. 2010, 46(6), 998-1007.

[7] TOGUN, N.K., BAYSEC, S. Prediction of torque and specific fuel consumption of a gasoline engine by using artificial neural networks. Applied Energy. 2010, 87, 349355.

[8] YIN, X., GE, A. A dynamic model of engine using neural network description. Vehicle Electronics Conference. 2001. 